\title{
Evaluation of Structural Properties of the Freeze-Thaw-Damaged RC Beam Members by Nonlinear Finite-Element Analysis
}

\author{
Hiroshi Hayashida \\ Civil Engineering Research Institute for Cold Region, Japan \\ Yasuhiko Sato and Tamon Ueda \\ Hokkaido University
}

\begin{abstract}
On RC beam members damaged by freeze-thaw cycles, the authors performed nonlinear finite-element analysis using distributed reinforcement and smeared cracking models, toward evaluating the structural properties of such RC beams. We then compared the analysis results to test results. The comparison found that the accuracy of analysis depends on the severity of concrete deterioration. It was clarified that the test values do not agree with the analysis values for RC beams that experience shear rigidity reduction from freezing damage to the compressive area or for RC beams that experience decreased rebar-concrete bonding strength from freezing damage to the tensile area. It was also clarified that the analysis may enable the evaluation of the rigidity to yield the failure mode and the maximum load for RC beams that have experienced no major decreases in shear rigidity and bonding strength.
\end{abstract}

Keywords: repeated freeze-thaw cycles, RC beam members, structural property, nonlinear finite-element analysis, shear rigidity, bonding strength.

\section{INTRODUCTION}

The authors have been working to develop a method for predicting the structural properties of reinforced concrete members that undergo freeze-thaw cycles. Hayashida, Sato, and Ueda (2012) conducted a static loading test of RC beam members under the parameters of the size and location of the freezing-damaged area, and they proved that the maximum load, ductility, and failure mode of the $\mathrm{RC}$ beam members depend on two parameters: the side on which the freezing-damaged area appears (compression vs. tension) and the size of the freezing-damaged area.

This paper focuses on nonlinear finite-element analysis, which previous research (Japan Society of Civil Engineers, 2006, 2009) has applied to evaluation of the properties of deteriorated concrete structures. Toward revealing the applicability and limitations of such structural property evaluation for freeze-thawdamaged reinforced concrete members, the present paper reports the results of nonlinear finite-element analysis of specimens tested by Hayashida et al.

\section{ANALYZED SPECIMENS}

This study analyzes the same specimens that were used in a study by Hayashida et al. (2012) Figure 1 shows the configuration, dimensions, and bar arrangement of a specimen. The specimen is a flexuralfailure-type RC beam member with two $\mathrm{D} 13$ main bars inside. The dimensions are $200 \mathrm{~mm} \times 200 \mathrm{~mm}$, and the span length is $1200 \mathrm{~mm}$. In categorizing the potentially freezing-damaged area into compression and tension sides, we assigned the deterioration penetration depths of 50,100 , and $150 \mathrm{~mm}$ from both side fibers. Table 1 shows the experimental variables and the test results of the specimens. In this table, the " $C$ " in the specimen names stands for "compression" and the $T$ stands for "tension", with each letters specifying the side that underwent concrete deterioration. " $\mathrm{N}$ " is the reference specimen, which was not subjected to any freeze-thaw action.

The tested specimen was pin supported, and the load was imposed only at the center of the span.

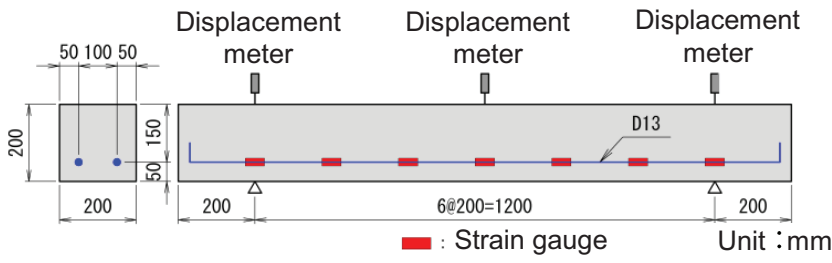

Figure 1. Configuration, dimensions, and bar arrangement of the specimen. 
Table 1. Specimen names and the tested and analyzed values.

\begin{tabular}{|c|c|c|c|c|c|c|c|c|}
\hline \multicolumn{3}{|c|}{ Specimen description } & \multicolumn{2}{|c|}{ Test } & \multicolumn{2}{|c|}{ Analysis } & \multicolumn{2}{|c|}{ Load ratio } \\
\hline Name & Deteriorated side & Deteriorated depth $(\mathrm{mm})$ & (a) $P_{\mathrm{y}}(\mathrm{kN})$ & (b) $P_{\mathrm{u}}(\mathrm{kN})$ & (c) $P_{y}(\mathrm{kN})$ & (d) $P_{\mathrm{u}}$ (kN) & c/a & $d / b$ \\
\hline $\mathrm{N}$ & - & - & 43.7 & 68.9 & 43.6 & 48.8 & $100 \%$ & $71 \%$ \\
\hline C5 & Compression side & 50 & 44.4 & 68.1 & 41.9 & 44.3 & $94 \%$ & $65 \%$ \\
\hline C10 & Compression side & 100 & 43.0 & 46.4 & 40.3 & 41.6 & $94 \%$ & $90 \%$ \\
\hline C15 & Compression side & 150 & - & 32.6 & 36.6 & 38.3 & - & $117 \%$ \\
\hline T10 & Tension side & 100 & 49.7 & 68.9 & 43.2 & 48.0 & $87 \%$ & $70 \%$ \\
\hline T15 & Tension side & 150 & 41.3 & 43.0 & 42.0 & 44.2 & $102 \%$ & $103 \%$ \\
\hline
\end{tabular}

$P_{\mathrm{y}}$ : yield load; $\mathrm{P}_{\mathrm{u}}$ : maximum load.

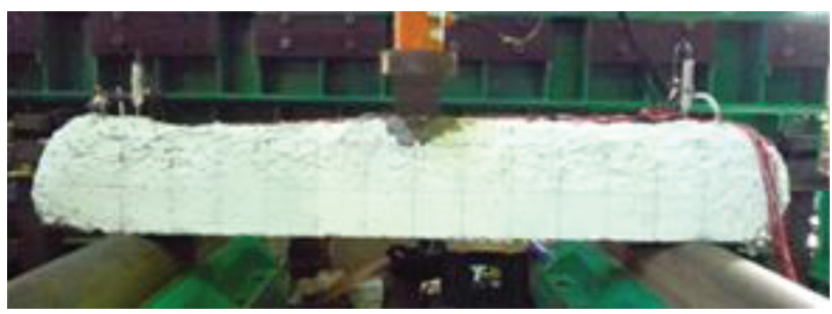

Photo 1. Loading test.

\section{OUTLINE OF FINITE-ELEMENT ANALYSIS}

\subsection{Analysis model}

For this study, we applied a versatile 2D nonlinear finiteelement analysis program called WCOMD. Figure 2 shows a model of finite elements, or divided elements. As repeated freeze-thaw cycles do not cause the specimen strength to deteriorate uniformly through each specimen, we modeled the whole span of the beam to reflect the various compressive strengths at various locations within the beam. The finite element in this study is an eight-node isoparametric plate stress element. The load was imposed perpendicularly at the center of the span $(0.1 \mathrm{~mm}$ per step). We also modeled loading and supporting plates that would restrain perpendicular deformation at the element's core point and that would avoid local element failure by stress concentration.

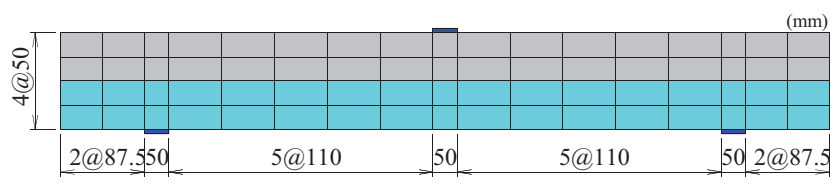

Figure 2. Finite-element model, with the divided elements.

\subsection{Modeling of cracks and rebar}

We modeled the concrete and rebar using reinforced concrete (RC) elements based on the models of smeared cracking and distributed reinforcement. Under our model, the volume of rebar in each individual RC element is expressed as a proportion of each element's total volume. Since the analysis assumes that no shear-reinforcing bars are installed, no elements were oriented perpendicularly and, thus, the volume of perpendicular rebar as a proportion of the element's total volume was always zero. In the model, the rebar-installed area (the RC zone, the blue in Figure 2) is expected to experience smeared cracking from bonding to the rebar, whereas the area without rebar (the no-RC zone, the gray in Figure 2) is not. We assumed that the RC zone of the main rebar extends $100 \mathrm{~mm}$ perpendicularly from the extreme tension fiber, which is twice the cover length and $200 \mathrm{~mm}$ horizontally to the back of the beam. It is noted that our analysis adopts the omnidirectional, nonperpendicular, fixed-crack model (Maekawa \& Fukuura, 1999), which reflects a model of smeared crack, quad-directional cracking.

\subsection{Material structure model}

The constitutive rule of reinforced concrete introduces a nonlinear constitutive rule that reflects the dependency on loading route developed by Okamura and Maekawa. The rule also reflects the tension stiffening effect, which comes from the bonding between concrete and rebar, the model of shear transfer on the crack surface and the lower compressive rigidity in the direction perpendicular to the cracks (Okamura \& Maekawa, 1990). Our analysis program needed a bonding parameter, $C$, to reflect the tension stiffening effect. In light of this, we assigned a default value of 0.4 to $C$ for all $\mathrm{RC}$ elements oriented parallel to the rebar. For the direction without rebar placement and for plain concrete elements in the compressive area, we computed the fracture energy, $G_{F}$, within the program (Equation (1)) and then assigned the bonding parameter that agrees with this fracture energy and element size.

$$
G_{F}=10\left(d_{\max }\right)^{1 / 3} \cdot f_{c}^{1 / 3}
$$

where dmax: maximum diameter of coarse aggregate (20 mm); $f_{c}^{\prime}$ : compressive strength.

The shear transfer coefficient is given the default value of 1.0 , which normally applies to concrete. We 
modeled loading and supporting plates using elastic material elements because plastic deformation was not confirmed during the test.

\subsection{Material properties of concrete and rebar}

The analyses needed to incorporate the material properties of concrete, for example, the compressive strength, given that freezing-damaged sizes and locations vary by specimen, each of which has irregular material properties. Accordingly, the analysis accounts for the spatial irregularities of those material properties using a lower compressive strength of concrete (Hashimoto, Morikawa, Saeki, \& Kobayashi, 2002). In our study, we measured the ultrasonic propagation velocity of deteriorated specimens at the positions shown in Figure 3. Also, we prepared a cylindrical specimen made from the same concrete as the tested RC beam and computed the correlation between the measured ultrasonic propagation velocity and the compressive strength (Figure 4), to estimate the compressive strengths at various locations on the specimen. From the individually computed compressive strengths, we assigned a compressive strength to each element. Figure 5 shows an example of the assigned compressive strength distribution. It is noted that the tensile strength and compressive peak strain of concrete were automatically assigned from compressive strengths within the program using the relative Equations (2) and (3) applied to concrete. It is also noted that this paper does not address whether the relations among concrete's compressive strength, tensile strength, and compressive peak strain remain the same regardless of concrete deterioration. We only focused on the applicability of the versatile analysis program; thus, we used the relational equation between dynamic properties under the condition of undeteriorated concrete.

$$
\begin{gathered}
f_{\mathrm{t}}=0.23 \cdot f_{\mathrm{c}}^{\prime 2 / 3} \\
\varepsilon_{\text {peak }}=140 \cdot f_{\mathrm{c}}^{\mathrm{\prime 1} / 2} \times 10^{-6},
\end{gathered}
$$

where, $f_{\mathrm{t}}$ : tensile strength, $\varepsilon_{\text {peak }}$ : compressive peak strain, and $f_{\mathrm{c}}^{\prime}$ : compressive strength.

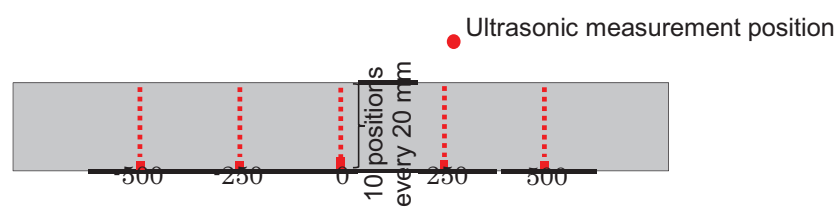

Figure 3. Measurement locations of ultrasonic propagation rate.

We assigned a compressive strength of $30.4 \mathrm{~N} /$ $\mathrm{mm}^{2}$ to undeteriorated specimen $N$, which is the compressive strength $\left(f_{c}^{\prime}\right)$ in the fourth week of concrete placement. For concrete tensile strength, we applied a value $30 \%$ smaller than the tensile strength estimated from tensile compressive strength under the JSCE Standard Specification for Concrete Structures (Japan Society of Civil Engineers, 2008), as drying shrinkage-causing initial stress may cause the tensile strength to be underestimated relative to that computed by the JSCE equation (Maekawa \& Fukuura, 1999) .

We assigned a yield strength of rebar $\left(f_{y}\right)$ of $361 \mathrm{~N} /$ $\mathrm{mm}^{2}$, specified in an inspection certificate, and an elastic coefficient of rebar $\left(E_{\mathrm{s}}\right)$ of $2.0 \times 10^{5} \mathrm{~N} / \mathrm{mm}^{2}$.

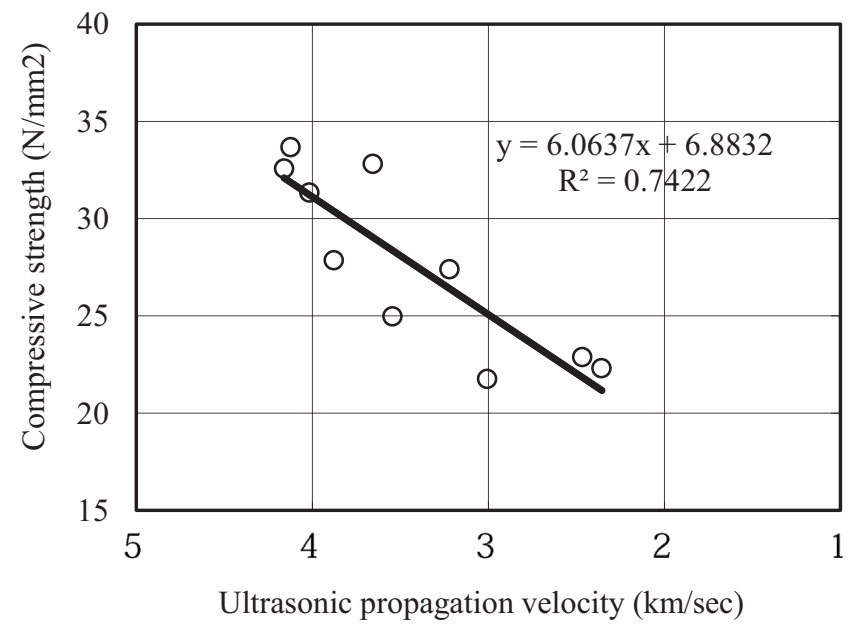

Figure 4. Ultrasonic propagation velocity vs. compressive strength.

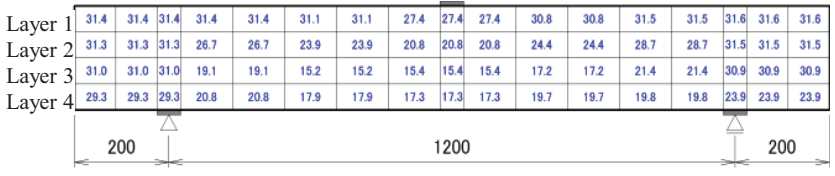

Figure 5. An example of compressive strength distribution (Specimen T10).

\subsection{Analysis cases}

For all the specimens except $N$, two conditions were given to the analysis (Table 2). In the first analysis, we assigned a compressive strength to each element, based on the ultrasonic propagation velocity (Case 1 in Figure 5). In the second analysis, we assigned the average values of compressive strength in the axial direction of the members in Layers I-IV (Case 2). For example, the compressive strengths of Specimen T10 are $30.2 \mathrm{~N} / \mathrm{mm}^{2}$ for Layer 1, $24.5 \mathrm{~N} / \mathrm{mm}^{2}$ for Layer 2, $17.5 \mathrm{~N} / \mathrm{mm}^{2}$ for Layer 3, and $18.9 \mathrm{~N} / \mathrm{mm}^{2}$ for Layer 4 . If every element is given a nonuniform strength, an element with lower strength may compress and soften quicker than the surrounding elements. Therefore, local increases in strain may cause load to decrease at an earlier stage, leading to underestimation of the maximum load of the materials. However, actual failure does not occur locally. Instead, it occurs in a wider area.

This is why we prepared two conditions of analysis. 
Table 2. Two analyses cases.

\begin{tabular}{cc}
\hline CASE 1 & $\begin{array}{r}\text { Based on ultrasonic propagation velocity results, } \\
\text { strength was nonuniform for every element }\end{array}$ \\
CASE 2 & $\begin{array}{r}\text { Strengths were averaged in individual layers in the } \\
\text { direction of the material axis }\end{array}$ \\
\hline
\end{tabular}

\section{ANALYSIS RESULTS AND DISCUSSION}

\subsection{Affect of the assigned compressive strength}

To investigate how the compressive strength affected the results, we conducted two conditions for analysis. We found that the results were roughly the same, and we only address Specimen C10.

Figure 6 plots load vs. deformation for $\mathrm{C} 10$ under the two cases. Case 1 has nonuniform compressive strength in each element, based on the ultrasonic propagation velocity, whereas Case 2 averages such compressive strengths. In terms of load vs. deformation, Case 2 has greater maximum load and deformation and is closer to the test value than Case 1. This is probably attributable to the fact that the averaged compressive strength actually slightly increases the strengths of the softened elements around the loading point. As the difference between the cases is not very large, we compare the Case 2 analysis results to the test results for discussion.

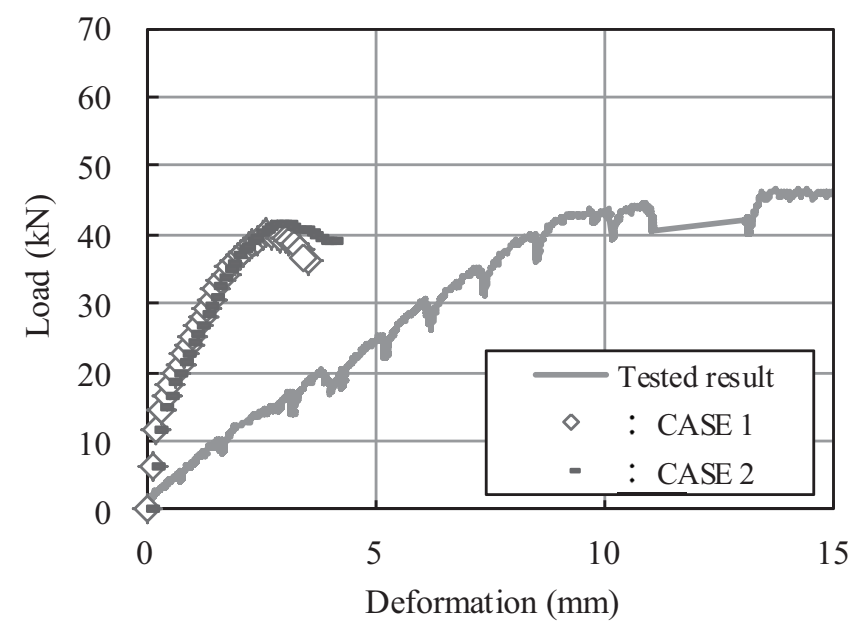

Figure 6. Specimen C10 loading and deformation relation.

\subsection{Comparison of tested and analyzed RC beam materials under frost damage}

we compared the tested and analyzed values in terms of failure mode, ductility, load-carrying capacity, and rebar strain distribution, for the purpose of summarizing how well the analysis reproduces the test values.

\subsubsection{Failure mode}

As shown in Figure 7, the analysis of all specimens indicates that elements around the loading point were compressed and failed, which led to smaller load. Between the test and analysis, the failure mode mostly agrees for specimens N, C5, and T10, but not for specimens C10, C15, and T15. C10 and C15 had failures of the stem concrete in the damaged area (diagonal compressive failure, Photo 2), and T15 had a wide shear crack (diagonal tensile failure).

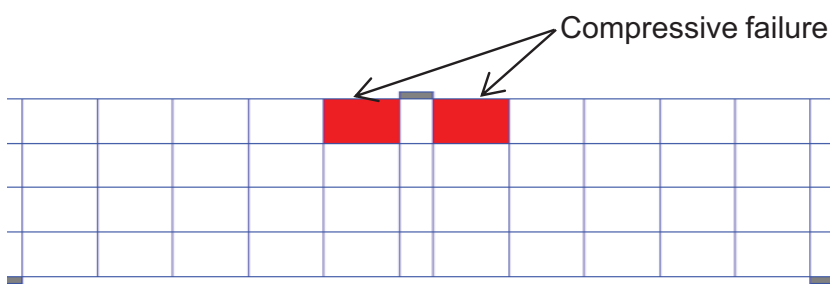

Figure 7. Damage at the ultimate stage.

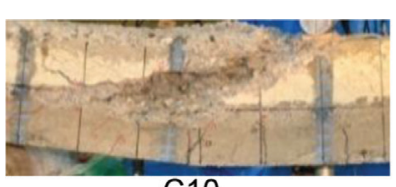

C10

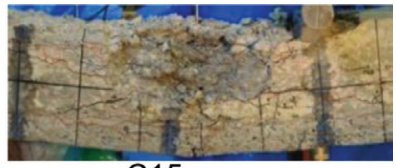

C15

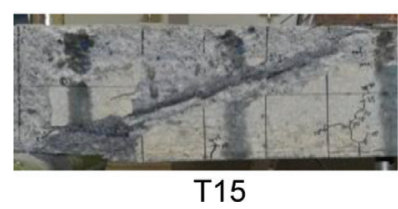

Photo 2. Specimens $\mathrm{C} 10, \mathrm{C} 15$, and $\mathrm{T} 15$ at the ultimate stage.

4.2.2 Ductility

For $\mathrm{N}$ and $\mathrm{T} 10$, the tested and analyzed values agreed in terms of the rigidity up to a member's yield point (Figure 8). For C5, the values look different, as the tested value shows a convex curve immediately after loading starts (Hasan, Okuyama, Sato, \& Ueda, 2003), whereas the analyzed one has a steeper gradient until cracks emerge. However, the gradients of the two results after cracks appear do not differ much, except immediately after the beginning of loading. For C10, C15, and T15, the analyzed gradients are notably greater than the tested ones. For C5, C10, and C15, the rigidity difference between tested and analyzed values increases with increase in the freezing penetration depth.

When we look at behaviors and after the member's yield point, the tested values fall into two categories: those with a yield plateau (C10, C5, T10) and those without a yield plateau (C10, C15, T15). For the yield plateau, as well as for the rigidity of concrete up to a member yield point, the analyzed and tested values agree only for $\mathrm{N}$ and T10.

It is noted that the length of the yield plateau in $\mathrm{N}$ and T10 is much shorter for the analyzed values than for the tested ones.

Also, all specimens show much smaller deformation at the maximum load in the analysis than in the test. This is attributed to the differences in rigidity and yield plateau length. 


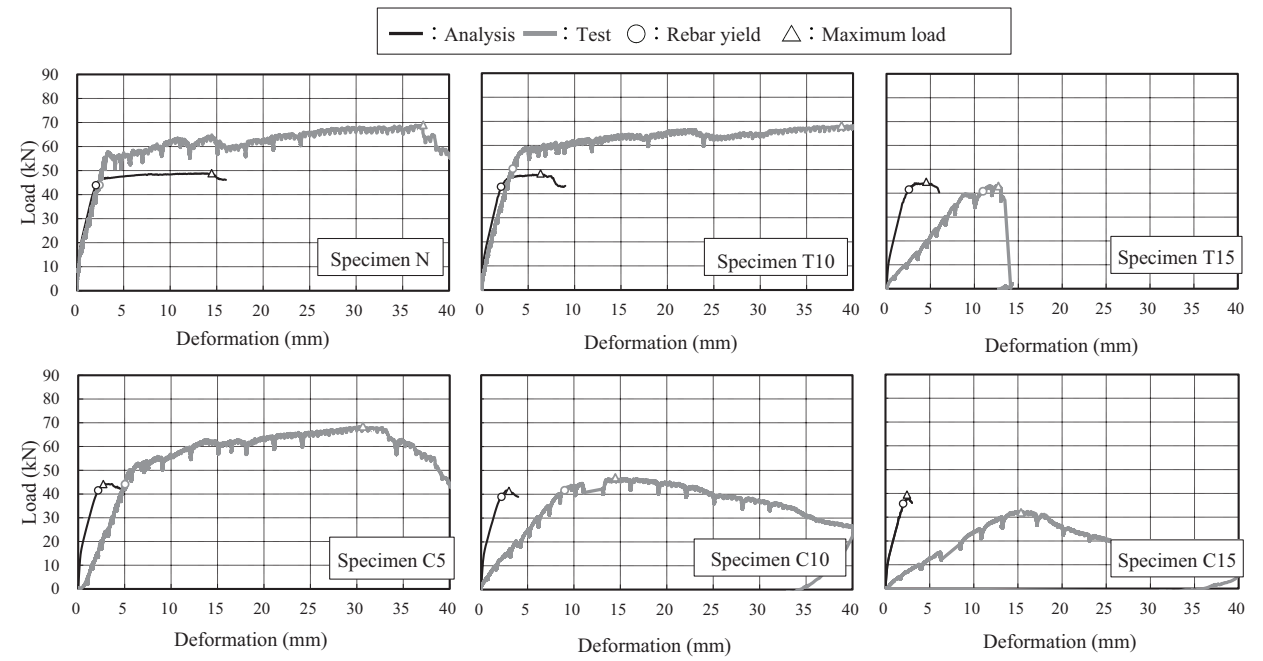

Figure 8. Comparison of tested and analyzed values for loading vs. deformation.

\subsubsection{Load-carrying capacity}

Regarding load-deformation relations for $N$, the tested load at and after the member yield point is greater than the analyzed load (Figure 8). This is probably because the tested specimens are pin supported (Photo 1), which generates a lateral restraining force in the perpendicular deformable area and seems to increase the rigidity. In light of this, we compare the ratio of tested to analyze yield load and maximum load.

The tested yield load is the load at $1800 \mu$ in the rebar strain gage at the center of the span, and the analyzed yield load is the load when the yield generated at the extreme tension fiber at the span center reaches the Gauss integration point at the bottom of the second element (black-bolded in Figure 9). Table 1 shows that the load ratios of the damaged specimens range from 87 to $102 \%$, which are roughly the same as those for $N$.

At the maximum load, C5 and T10 with yield plateaus show the values similar to those for $N$, whereas the maximum loads for C10, C15, and T15 without yield plateaus are greater than those for $N$.

\subsubsection{Rebar strain distribution}

Figure 10 shows rebar strain distributions at the $30 \mathrm{kN}$ load for the tested and analyzed specimens. It is noted that the analyzed rebar strain is the strain at the Gauss integration point at the upper center of the extreme tension fiber element, which is plotted in the direction of the member axis. For example, the rebar strain at the center of the span is the green circle in Figure 9, strain at the Gauss integration point.

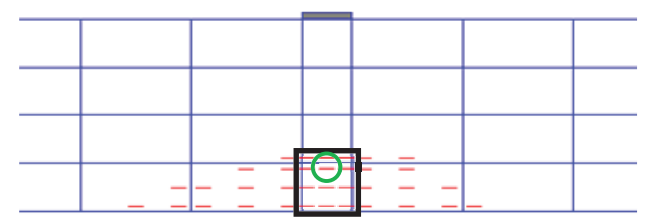

Figure 9. Rebar yield at the yield load (C10). a) Series of deterioration on the compression side (Series C)

The upper chart in Figure 10 shows that the tested rebar strains for $\mathrm{C} 5$ and $\mathrm{C} 10$ are similar to those for $N$. From this, it is considered that the bonding strengths of the Series C specimens do not decrease. In C15, a large strain spreads beyond $N$ to areas adjacent to the pin-supported points. This is attributed to the significantly lower bonding strength between rebar and concrete.

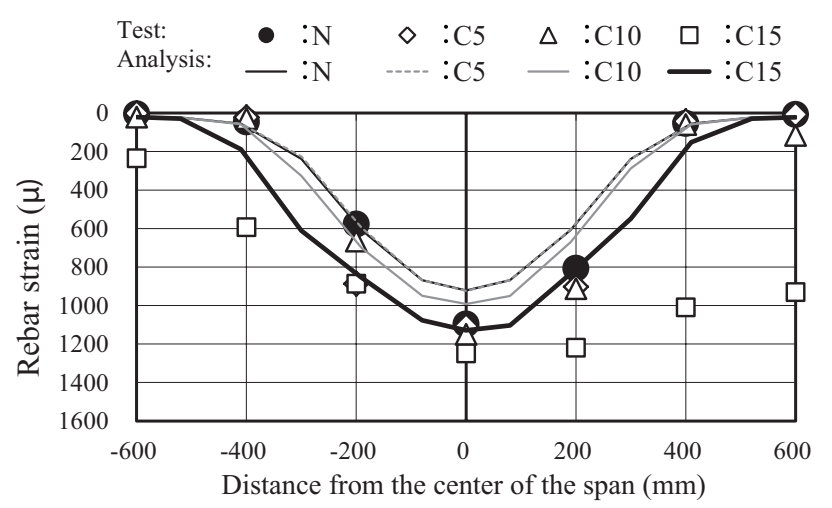

(Series of Deterioration on the Compression Side)

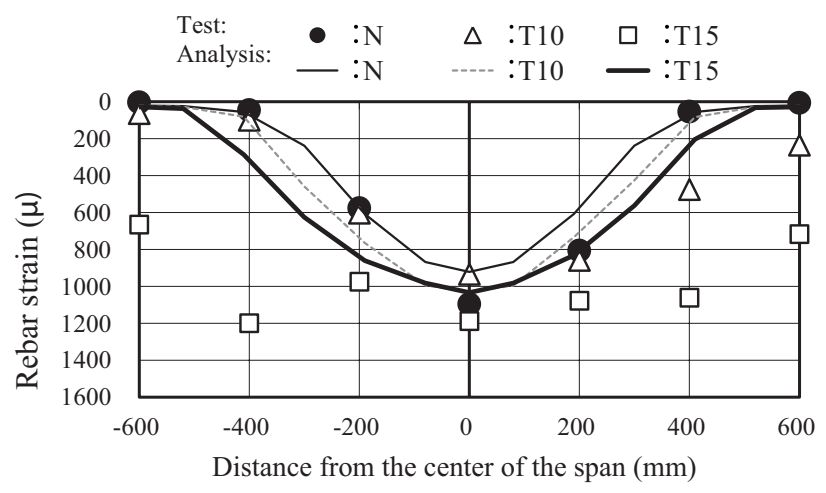

(Series of Deterioration on the Tension Side)

Figure 10. Rebar strain distribution at $30 \mathrm{kN}$ load. 
In the analysis, the rebar strain for $\mathrm{C} 5$ is similar to that of $N$, whereas the strains for $\mathrm{C} 10$ and $\mathrm{C} 15$ are greater than that of $N$, and the difference increases with increase in the freezing-damage penetration depth.

In comparing the tested and analyzed values of the leftside span which were relatively uniformly measured, $N$ and T10 agree at all locations, except at the center of the span. C5 also agrees in overall distribution, except at $-200 \mathrm{~mm}$. However, C15 shows a greater tested strain than analyzed strain. It is considered that the bonding strengths decrease more in the test than the analysis.

\section{b) Series of deterioration on the tension side (Series T)}

The lower chart in Figure 10 shows that the tested rebar strain for T10 exceeds that for $N$ at 400 and $600 \mathrm{~mm}$ on the right-side span, but that the difference is not very significant. On the left-side span, the strain distributions of T10 and $N$ are similar; thus, it is considered that bonding strengths at the $30 \mathrm{kN}$ load are not greatly decreased. However, the distribution of T15 shows that a large strain spreads beyond $N$ to areas adjacent to the pin-supported points and bonding strength decreases greatly.

In the analysis, the rebar strains for T10 and T15 are greater than those for $N$, and the difference increases with increase in the freezing penetration depth. This trend is similar to $\mathrm{C} 10$ and $\mathrm{C} 15$.

The tested and analyzed values for T10 agree at all locations, except at 400 and $600 \mathrm{~mm}$ on the right span, at which the tested values are slightly greater than the analyzed ones. From this, it is safe to consider that the bonding strength of T10 does not decrease significantly, given that the tested values generally agree with the analyzed ones that assume complete bonding between rebar and concrete. However, the tested T15 shows greater strain than the analyzed one; thus, it is considered that bonding strength decreases more in the test than the analysis.

\subsubsection{Discussion on the difference between test and analysis}

Photo 3 shows the damage to the tested C10. Several shear cracks were generated in the freezing-damaged compressive area of the tested C10 during loading. As deformation increased, those shear cracks widened and increased in number, the stem concrete around the cracks was crushed and the load decreased moderately. However, the analyzed C10 did not show such phenomena. From this, the rigidity difference between the tested and analyzed C10 is regarded as being attributable to the decrease in shear rigidity caused by the shear crack development. Because the shear rigidity of the compressive area decreased greatly, the tested C10 showed different behavior from analysis, leading to the differences in rigidity, failure mode, and maximum load.

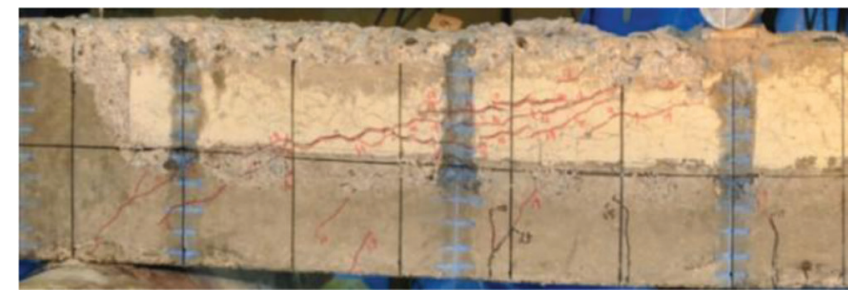

Photo 3. Damage at the maximum load (C10).

C15 and T15 evidently show lower bonding strengths than the rebar strains referred to in (4). It is considered that T15's lower bonding strengths caused the difference between the tested behavior and the analyzed behavior, resulting in differences in rigidity, failure mode, and maximum load, and that C15's lower bonding strengths plus lower shear rigidity were factors in the greater decrease in rigidity and maximum load than that for $\mathrm{C} 10$, which further increased the difference between the tested and analyzed values.

For RC beams with relatively small decrease in the shear rigidity of the compression area or lower bonding strength of the tensile area, such as C5 and T10, nonlinear finite-element analysis may be able to relatively and appropriately evaluate the beams' rigidity, failure mode, and maximum load to yield, as long as the analysis accounts for spatial irregularity by incorporating a reduced concrete compression strength.

\section{CONCLUSION}

In this study, we performed tests and versatile program analyses to evaluate the structural properties of freeze-thaw-damaged RC beams. Then we compared the two results toward clarifying the applicability of nonlinear finite-element analysis using the distributed rebar model and the smeared cracking model. The findings are as follows:

(1) The behavior of freezing-damaged RC beam members is not precisely reproduced when such beams' compression areas are severely damaged, and they decrease in shear rigidity, or when the rebar-concrete bonding strength decreases.

(2) Nonlinear finite-element analysis may be usable for evaluating rigidity, failure mode, and maximum load of such RC beams relatively precise, provided that the beams do not experience decrease in shear rigidity in the compression area or in bonding strengths in the tensile area.

It should be noted that these findings were obtained only for tests using RC beams with relatively severe freezing damage and without shear reinforcing bars, and that there are no data on concrete strain, which is 
normally essential in comparing test values to analyze values. In light of this, further studies are required to establish a quantitative method for evaluating freezingdamaged concrete materials through additional tests that address the matters noted above.

Finally, this study is one of the achievements of the Sub-committee for Evaluating Damaged Concrete's Structural Properties: Concrete Study Committee of the Civil Engineering Association of Hokkaido.

\section{REFERENCES}

Hasan, M., Okuyama, H., Sato, Y., \& Ueda, T. (2003). Stress-strain model of concrete damaged by freezing and thawing cycles. Journal of Advanced Concrete Technology, 2(1), 89-99.

Hashimoto, W., Morikawa, H., Saeki, K., \& Kobayashi, H. (2002). Shear resisting mechanism of RC members considering concrete strength distribution. Proceedings of the Japan Concrete Institute, 24(2), 925-930.

Hayashida, H., Sato, Y., \& Ueda, T. (2012). Failure behavior of RC beams in relation to the extent and location of frost damage. In 3rd International Conference on the Durability of Concrete Structures (ICDCS2012), PER1/1-1/7, 2012, Belfast, Northern Ireland.

Japan Society of Civil Engineers. (2006). Performance of concrete structures with material deterioration. Concrete Engineering Series No. 71. Yotsuya, Japan: JSCE.

Japan Society of Civil Engineers. (2008). Standard specifications for concrete structures 2007: "Design". Yotsuya, Japan: JSCE.

Japan Society of Civil Engineers. (2009).

Performance of concrete structures with material deterioration 2. Concrete Engineering Series No. 85. Yotsuya, Japan: JSCE.

Maekawa, K., \& Fukuura, N. (1999) Verification of the spatially averaged constitutive model with multidirectional cracks on the behavior of RC structure. Proceedings of Japan Society of Civil Engineers, 45(634), 209-225.

Okamura, H., \& Maekawa, K. (1990). Nonlinear analysis and constitutive models of reinforced concrete. Tokyo, Japan: Gihodo Shuppan Co., Ltd. 